\title{
Zero products preserving maps from the Fourier algebra of amenable groups
}

\author{
Jafar Soltani Farsani
}

\begin{abstract}
Let $G$ be a locally compact amenable group. The goal of this paper is to investigate the problem of surjective zero products preserving maps from the Fourier algebra of $G$ into a completely contractive Banach algebra. We show that if $B$ is a completely contractive Banach algebra which is faithful and factors weakly, then every surjective completely bounded linear map from $A(G)$ into $B$ which preserves zero products is a weighted homomorphism. Moreover an equivalent condition is given for such a map to be a homomorphism. In particular, this result implies that if $B$ is a commutative $C^{*}$-algebra or a matrix space and $T: A(G) \rightarrow B$ is a continuous surjective linear map which preserves zero products, then $T$ is a weighted homomorphism and there is an equivalent condition for $T$ to be a homomorphism.
\end{abstract}

\section{Introduction}

Let $A$ and $B$ be two Banach algebras. A linear operator $T: A \rightarrow B$ is said to preserve zero products if it has the following property

$$
\forall a, b \in A a b=0 \Rightarrow T(a) T(b)=0 .
$$

Obviously if $T$ is a homomorphism, then it preserves the zero products. The question that might be challenging is the converse, i.e, if $T$ is a zero products preserving map, then under what conditions $T$ is a homomorphism? In many cases,

Received by the editors in January 2013 - In revised form in November 2013.

Communicated by A. Valette.

2010 Mathematics Subject Classification : Primary 47B48; Secondary 43A30.

Key words and phrases : locally compact group, amenable group, Fourier algebra, operator spaces, set of spectral synthesis, Weighted homomorphism. 
being homomorphism is strong to be proven. Hence the question can be modified by replacing homomorphism with weighted homomorphism.

In prior to extending this question to general Banach algebras, it was a challenging question for different classes of Banach algebras. The zero products preserving maps in the case $A$ and $B$ are function algebras are usually called the separating maps or Lamperti operators. For more information about the separating maps between function algebras we refer the reader to [4]. The problem was investigated by $S$. Banach for the case $A=B=L^{p}[0,1]$, for $p \neq 2$ in [3]. It has also been considered for group algebras in [11] and for operator algebras. In the latter case, the reader is referred to $[5,9,14,15,16,18,21]$. The problem of continuous surjective zero products preserving maps was generalized to arbitrary Banach algebras by J. Alaminos, M. Brešar, J. Extremera and A. R. Viellena [2]. They introduced a property called the property $(\mathbb{B})$. It was shown that this property is of great importance in handling the zero products preserving maps.

In this paper, we consider completely bounded surjective linear maps from the Fourier algebra of a locally compact amenable group into a completely contractive Banach algebra which is faithful and factors weakly. We show that if such a map preserves zero products then it is a weighted homomorphism and being homomorphism is equivalent to a milder condition.

Our result in particular shows that if $B$ is a commutative $C^{*}$-algebra or a matrix space, then every continuous surjective zero products preserving map $T: A(G) \rightarrow B$ is a weighted homomorphism where $G$ is an amenable group. It also provides us an equivalent condition for $T$ to be a homomorphism. Our results also imply that if $G_{1}$ and $G_{2}$ are two amenable groups, then every completely bounded surjective linear map $T: A\left(G_{1}\right) \rightarrow A\left(G_{2}\right)$ which preserves zero products is of the form $T=\tau \Phi$. Where $\Phi: A\left(G_{1}\right) \rightarrow A\left(G_{2}\right)$ is a homomorphism and $\tau$ is an invertible element in the Fourier-Stieltjes algebra of $G_{2}$.

The key tool in our approach is to define a condition for a Banach algebra with an operator space structure which is similar to the property $(\mathbb{B})$ and we call it the property $(\mathbb{B})$ completely. We show that the Fourier algebra of every locally compact amenable group has the property $(\mathbb{B})$ completely.

\section{Preliminaries}

Let $G$ be a locally compact group and suppose that $\pi: G \rightarrow B\left(H_{\pi}\right)$ is a representation of $G$. For each $\xi, \eta \in H_{\pi}$, the map $\pi_{\xi, \eta}: G \rightarrow \mathbb{C}$ is defined with

$$
\pi_{\xi, \eta}(x)=<\pi(x) \xi, \eta>
$$

The Fourier-Stieltjes algebra of $G$ is defined by

$$
B(G)=\left\{\pi_{\xi, \eta}: \xi, \eta \in H_{\pi}, \pi \text { is a unitary representation of } G\right\} .
$$

It is well-known that $B(G)$ is the dual of the group $C^{*}$-algebra of $G$. The closure of $B(G) \cap C_{c}(G)$ in $B(G)$ is called the Fourier algebra and is denoted by $A(G)$. Note that $A(G)$ is a two sided ideal of $B(G)$. 
A locally compact group $G$ is called amenable if there is a left invariant mean on $L^{\infty}(G)$, i.e, there is a bounded functional $m$ on $L^{\infty}(G)$ such that

$$
\|m\|=m(1)=1 .
$$

and in addition

$$
\forall x \in G, \forall f \in L^{\infty}(G), \quad m\left(L_{x} f\right)=m(f) .
$$

Where $L_{x} f: G \rightarrow G$ is defined with

$$
L_{x} f(y)=f\left(x^{-1} y\right) \quad \forall y \in G .
$$

The following Theorem which is proven in [19, Theorem 10.4] gives an equivalent condition for a locally compact group $G$ to be amenable.

Theorem 2.1. Let $G$ be a locally compact group. $G$ is amenable if and only if $A(G)$ has a bounded approximate identity.

Theorem 2.2 ([19]). Let $G$ be a locally compact amenable group. The multiplier algebra of $A(G)$ is $B(G)$.

A closed subset $E$ of $G$ is said to be a set of spectral synthesis for $A(G)$ if for each $f \in A(G)$ which vanishes on $E$ and for each $\epsilon>0$ there exists $\varphi \in A(G)$ the support of which is compact and disjoint from $E$ and

$$
\|f-\varphi\|<\epsilon .
$$

Since the maximal ideal space of $A(G)$ is $G$ and the Gelfand transformation is the inclusion map from $A(G)$ into $C_{0}(G)$, this definition is just the standard definition of a set of spectral synthesis for a commutative semisimple regular Banach algebra.

Theorem 2.3 ([13]). Let $G$ be a locally compact group. Every closed amenable subgroup of $G$ is a set of spectral synthesis for $A(G)$.

Let $V$ and $W$ be two operator spaces and $\varphi: V \rightarrow W$ a linear mapping. the $n^{\text {th }}$ amplification of $\varphi$ is a linear map $\varphi_{n}: M_{n}(V) \rightarrow M_{n}(W)$ which is defined by

$$
\varphi_{n}(v)=\left[\varphi\left(v_{i j}\right)\right]
$$

for all $v=\left[v_{i j}\right] \in M_{n}(V)$. The completely bounded norm of $\varphi$ is defined by

$$
\|\varphi\|_{c b}=\sup \left\{\left\|\varphi_{n}\right\|: n \in \mathbb{N}\right\} .
$$

We say that $\varphi$ is completely bounded if $\|\varphi\|_{c b}<\infty$. Let $X$ be another operator space. Suppose that $\varphi: V \times W \rightarrow X$ is a bilinear map. For each $p, q \in \mathbb{N}$, the bilinear map $\varphi_{p ; q}: M_{p}(V) \times M_{q}(W) \rightarrow M_{p \times q}(X)$ is determined by

$$
\varphi_{p ; q}(v, w)=\left[\varphi\left(v_{i j}, w_{k l}\right)\right] .
$$

The completely bounded norm of $\varphi$ is defined by

$$
\begin{aligned}
\|\varphi\|_{c b} & =\sup \left\{\left\|\varphi_{p ; q}\right\|: p, q \in \mathbb{N}\right\} \\
& =\sup \left\{\left\|\varphi_{p ; p}\right\|: p \in \mathbb{N}\right\} .
\end{aligned}
$$

We say that $\varphi$ is completely bounded if $\|\varphi\|_{c b}<\infty$. 


\section{Fourier algebra of locally compact amenable groups and the property $(\mathbb{B})$ completely}

In this section we prove that for every locally compact group $G, A(G)$ has a property called the property $(\mathbb{B})$ completely. As mentioned before, this property is important in order to determine weighted homomorphisms and homomorphisms among the zero products preserving maps.

This is well-known that $A(G)$ is the predual of the group von Neumann algebra of $G$ as a Banach space. Hence it inherits the operator space structure of $V N(G)^{*}$. Consider $A(G)$ as an operator space with this structure.

Theorem 3.1 ([12]). Let $G$ be a locally compact group. Then

$$
A(G) \widehat{\otimes} A(G) \cong A(G \times G) \text { completely isometrically. }
$$

Where $\widehat{\otimes}$ stands for the operator space projective tensor product. The complete isomorphism is given by considering $a \otimes b$ as a function on $G \times G$ with

$$
\forall g_{1}, g_{2} \in G(a \otimes b)\left(g_{1}, g_{2}\right)=a\left(g_{1}\right) b\left(g_{2}\right)
$$

For more information on the operator space projective tensor product see chapter 7 in [12].

The following definition first appeared in [1].

Definition 3.2. Let $A$ be a Banach algebra. We say that $A$ has the property $(\mathbb{B})$ if for every Banach space $X$ and each continuous bilinear map $\varphi: A \times A \rightarrow X$ with the property,

$$
a, b \in A \quad a b=0 \Rightarrow \varphi(a, b)=0
$$

we can infer that

$$
\forall a, b, c \in A \varphi(a b, c)=\varphi(a, b c) .
$$

We modify this definition by adding an operator space structure to the Banach algebra and confining ourselves to the completely bounded bilinear maps.

Definition 3.3. Let $A$ be a Banach algebra with an operator space structure. We say that $A$ has the property $(\mathbb{B})$ completely if for every operator space $X$ and each completely bounded bilinear map $\varphi: A \times A \rightarrow X$ with the property,

$$
a, b \in A a b=0 \Rightarrow \varphi(a, b)=0
$$

it satisfies in the following,

$$
\forall a, b, c \in A \varphi(a b, c)=\varphi(a, b c) .
$$

Obviously if $A$ is a Banach algebra with an operator space structure which satisfies in the property $(\mathbb{B})$, then it satisfies in the property $(\mathbb{B})$ completely. 
Theorem 3.4. Let $G$ be a locally compact amenable group. Then $A(G)$ has the property (B) completely.

Proof. Let $X$ be an operator space and $\varphi: A(G) \times A(G) \rightarrow X$ a completely bounded bilinear map with the property,

$$
a, b \in A a b=0 \Rightarrow \varphi(a, b)=0 .
$$

$\varphi$ gives rise to a completely bounded linear map $\tilde{\varphi}: A(G) \widehat{\otimes} A(G) \rightarrow X$ which is given by

$$
\tilde{\varphi}(a \otimes b)=\varphi(a, b) .
$$

By Theorem 3.1, For each $a, b \in A(G), a \otimes b$ as a completely bounded bilinear map is defined by

$$
a \otimes b(x, y)=a(x) b(y) .
$$

We view $\tilde{\varphi}$ as a linear map on $A(G \times G)$. Let's define

$$
H=\{(g, g): g \in G\} .
$$

This is a closed amenable subgroup of $G \times G$. Hence by Theorem 2.3, $\mathrm{H}$ is a set of spectral synthesis for $A(G \times G)$. Let $I_{0}(H)$ denote the set of all elements of $A(G \times G)$ with compact support which is disjoint from $H$. By the definition of spectral synthesis, $I_{0}(H)$ is contained in every closed ideal of $A(G)$ the hull of which is $H$. Where for a subset $I$, its hull is defined with:

$$
\operatorname{hull}(I)=h(I)=\{(s, t) \in G \times G: u(s, t)=0 \forall u \in I\} .
$$

We also define the following subspace of $A(G \times G)$,

$$
J=\operatorname{span}\{a \otimes b: a, b \text { have compact supports and supp } a \cap \operatorname{supp} b=\varnothing\} .
$$

Note that $\bar{J}$ is an ideal of $A(G \times G)$. Moreover, this can be checked, using regularity of $A(G)$ that $h(\bar{J})=h(J)=H$. So $I_{0}(H) \subseteq \bar{J}$. It is clear that $J \subseteq I_{0}(H)$. Hence

$$
\bar{J}=\overline{I_{0}(H)} \text {. }
$$

If $I(H)$ is the set of all elements in $A(G \times G)$ which vanish on $\mathrm{H}, h(I(H))=H$. Hence (again using the spectral synthesis), $I(H)=\overline{I_{0}(H)}=\bar{J}$. Now we have

$$
a, b \in A(G) \text { supp } a \cap \operatorname{supp} b=\varnothing \Rightarrow a b=0 \Rightarrow \tilde{\varphi}(a \otimes b)=\varphi(a, b)=0 .
$$

Hence $\tilde{\varphi}$ vanishes on $J$. Continuity of $\tilde{\varphi}$ implies that

$$
\forall \alpha \in I(H) \quad \tilde{\varphi}(\alpha)=0 .
$$

Let $a, b, c \in A(G)$. For each $g \in G$ we can write

$$
(a b \otimes c-a \otimes b c)(g, g)=(a b c-a b c)(g)=0 .
$$

So $a b \otimes c-a \otimes b c \in I(H)$. Now by (3.1) we have

$$
\forall a, b, c \in A(G) \quad \tilde{\varphi}(a b \otimes c)=\tilde{\varphi}(a \otimes b c)
$$

Or equivalently

$$
\forall a, b, c \in A(G) \varphi(a b, c)=\varphi(a, b c) .
$$

It completes the proof. 


\section{Completely bounded surjective zero products preserving maps from Fourier algebras}

In this section, we show how the property $(\mathbb{B})$ completely can be applied to determine weighted homomorphisms and homomorphisms among the completely bounded linear maps which are surjective and preserve zero products. Let $A$ be a Banach algebra. $A$ is said to be faithful if

$$
\{a \in A: a A=A a=\{0\}\}=\{0\}
$$

Obviously any Banach algebra with an approximate identity is faithful. We say that $A$ factors weakly if $A=A^{2}$. By Cohen's factorization theorem every Banach algebra with a bounded approximate identity factors weakly. A centralizer on $A$ is a linear operator $W: A \rightarrow A$ satisfying

$$
W(a b)=W(a) b=a W(b) .
$$

If $A$ is faithful, then it is easy to check that

$$
\Gamma(A) \cong Z(M(A))
$$

Where $Z(M(A))$ stands for the center of the multiplier algebra of $A$ and $\Gamma(A)$ is the space of all centralizers on $A$.

Let $A$ and $B$ be two Banach algebras. A map $T: A \rightarrow B$ is called a weighted homomorphism if there is a homomorphism $\Phi: A \rightarrow B$ and an invertible centralizer $W \in \Gamma(B)$ such that $T=W \Phi$.

Definition 4.1. Let $A$ be an associative algebra over complex numbers. We call $A$ a completely contractive Banach algebra if $A$ is a complete operator space and the multiplication

$$
m: A \times A \rightarrow A
$$

is a completely contractive bilinear mapping. Equivalently for $m, n \in \mathbb{N}$ and for all $a=\left[a_{i j}\right] \in M_{m}(A), b=\left[b_{k l}\right] \in M_{n}(A)$ we should have,

$$
\left\|\left[a_{i j} b_{k l}\right]\right\| \leq\|a\|\|b\| .
$$

$C^{*}$-algebras and Fourier algebra of any locally compact group are examples of completely contractive Banach algebras. For more information see chapter 16 in [12].

Lemma 4.2. Let $A$ be a Banach algebra with an operator space structure having the property $(\mathbb{B})$ completely and suppose that $B$ is a completely contractive Banach algebra. If $T: A \rightarrow B$ is a completely bounded linear map preserving zero products, then

$$
T(a b) T(c)=T(a) T(b c) \quad \forall a, b, c \in A .
$$


Proof. Define a bilinear map $\varphi: A \times A \rightarrow B$ by

$$
\varphi(a, b)=T(a) T(b) \quad(a, b \in A) .
$$

If $a, b \in A$ and $a b=0$, then $\varphi(a, b)=0$. For each $n \in \mathbb{N}$ and each $a, b \in M_{n}(A)$ we have,

$$
\begin{aligned}
\left\|\varphi_{n ; n}(a, b)\right\| & =\left\|\left[\varphi\left(a_{i j}, b_{k l}\right)\right]\right\| \\
& =\left\|\left[T\left(a_{i j}\right) T\left(b_{k l}\right)\right]\right\| \\
& \leq\left\|\left[T\left(a_{i j}\right)\right]\right\|\left\|\left[T\left(b_{k l}\right)\right]\right\| \\
& \leq\|T\|_{c b}^{2}\|a\|\|b\| .
\end{aligned}
$$

It implies that

$$
\|\varphi\|_{c b} \leq\|T\|_{c b}^{2} .
$$

Hence $\varphi$ is completely bounded. Since $A$ has the property $(\mathbb{B})$ completely, We obtain the required result.

Corollary 4.3. Let $A$ be a Banach algebra with an operator space structure which has the property $(\mathbb{B})$ completely. Suppose that $B$ is a completely contractive Banach algebra. If $T: A \rightarrow B$ is a completely bounded linear map preserving zero products, then the following assertions are equivalent:

(i) $T$ is a homomorphism.

(ii) A has an approximate identity $\left(\rho_{i}\right)_{i \in I}$ such that $\left(T\left(\rho_{i}\right)\right)_{i \in I}$ is an approximate identity for $B$.

Proof. Obviously $(i)$ implies (ii). To prove the converse, let $\left(\rho_{i}\right)_{i \in I}$ be an approximate identity for $A$ such that $\left(T\left(\rho_{i}\right)\right)_{i \in I}$ is an approximate identity for $B$. According to Lemma 4.2 , for each $i \in I$ we have

$$
T(a b) T\left(\rho_{i}\right)=T(a) T\left(b \rho_{i}\right) \quad \forall a, b \in A .
$$

Passing through limit, we get

$$
T(a b)=T(a) T(b) \quad \forall a, b \in A .
$$

Lemma 4.2 enables us to prove the next two Theorems the idea of which come from [2, Theorem 3.6] and [2, Theorem 3.8] respectively. While those theorems are in the category of Banach algebras with the property $(\mathbb{B})$, our theorems are concerned with Banach algebras which have an operator spaces structure with the property $(\mathbb{B})$ completely.

Theorem 4.4. Let $A$ be a Banach algebra with an operator space structure having the property $(\mathrm{B})$ completely and having a left approximate identity. Suppose that $B$ is a completely contractive Banach algebra. If $T$ is a completely bounded linear map which preserves zero products, then there is a right ideal $I_{V}$ of $B$ containing $T\left(A^{2}\right)$ and a linear operator $V: I_{V} \rightarrow B$ such that

$$
V(u v)=V(u) v, \quad V(z w)=z V(w) \quad\left(u \in I_{V}, v \in B, w \in \operatorname{alg} T\left(A^{2}\right), z \in \operatorname{alg} T(A)\right)
$$


and

$$
V(T(a b))=T(a) T(b) \quad(a, b \in A) .
$$

Furthermore the following assertions hold:

(i) If $B$ is faithful and $\overline{T(A)}=B$, then there is a two sided ideal $I_{W}$ of $B$ containing $T\left(A^{2}\right)$ and a closed linear operator $W: I_{W} \rightarrow B$ such that $W$ coincides with $V$ on $T\left(A_{2}\right)$ and

$$
W(u v)=W(u) v \text { and } W(v u)=v W(u)\left(u \in I_{W}, v \in B\right) .
$$

Moreover, if $B$ is essential, then $\overline{W\left(I_{W}\right)}=B$ and $W$ is injective.

(ii) If $B$ is faithful and factors weakly, and $T(A)=B$, then the operator $W$ given in $(i)$ is invertible and there is a continuous epimorphism $\Phi: A \rightarrow I_{W}$ such that $T=W \Phi$.

Proof. Suppose that $\left(\rho_{i}\right)_{i \in I}$ is a left approximate identity for $A$. Let

$$
I_{V}=\left\{u \in B:\left(T\left(\rho_{i}\right) u\right)_{i \in I} \text { converges }\right\}
$$

and define $V: I_{V} \rightarrow B$ by

$$
V(u)=\lim _{i \in I} T\left(\rho_{i}\right) u \quad\left(u \in I_{V}\right) .
$$

It is clear that $I_{V}$ is a right ideal of $B$ and that

$$
V(u v)=V(u) v \quad\left(u \in I_{V}, v \in B\right) .
$$

By Lemma 4.2 we get

$$
T(a) T(b)=\lim _{i \in I} T\left(\rho_{i} a\right) T(b)=\lim _{i \in I} T\left(\rho_{i}\right) T(a b) \quad(a, b \in A) .
$$

It shows that $T\left(A^{2}\right) \subset I_{V}$ and

$$
V(T(a b))=T(a) T(b) \quad(a, b \in A) .
$$

Note that Lemma 4.2 shows that $T(A) T\left(A^{2}\right)=T\left(A^{2}\right) T(A) \subset I_{V}$ and for all $a_{1}, \ldots, a_{n}, b, c \in A$ we have

$$
\begin{aligned}
V\left(T\left(a_{1}\right) \ldots T\left(a_{n}\right) T(b c)\right) & =\lim _{i \in I} T\left(\rho_{i}\right) T\left(a_{1}\right) \ldots T\left(a_{n}\right) T(b c) \\
& =\lim _{i \in I} T\left(\rho_{i} a_{1}\right) \ldots T\left(a_{n}\right) T(b) T(c) \\
& =T\left(a_{1}\right) \ldots T\left(a_{n}\right) T(b) T(c) \\
& =T\left(a_{1}\right) \ldots T\left(a_{n}\right) V(T(b c)) .
\end{aligned}
$$

Hence we infer that

$$
V(z w)=z V(w) \quad\left(z \in \operatorname{alg} T(A), w \in \operatorname{alg} T\left(A^{2}\right)\right) .
$$

Now suppose that $B$ is faithful and that $\overline{T(A)}=B$. Since $A$ has a left approximate identity, it follows that $A^{2}$ is dense in $A$, and since $T(A)$ is dense in $B$, so is $\operatorname{algT}\left(A^{2}\right)$. Using (4.1), (4.4) and [2, Lemma 3.5], the restriction of the operator 
$V$ to $\operatorname{alg} T\left(A^{2}\right)$ is closable and the closure $W: I_{W} \rightarrow B$ of the operator has the property given in (i). From (4.3) we see that $T(A)^{2} \subset W\left(T\left(A^{2}\right)\right) \subset W\left(I_{W}\right)$ and so $\overline{B^{2}} \subset \overline{W\left(I_{W}\right)}$. This shows that $\overline{W\left(I_{W}\right)}=B$ if $B$ is essential. Note that $W$ is injective. To show this, let $u \in k e r W$ and $v \in I_{W}$ we have

$$
u W(v)=W(u v)=W(u) v=0,
$$

similarly $W(v) u=0$. Since $\overline{W\left(I_{W}\right)}=B$ and $B$ is faithful, we deduce that $u=0$. Next suppose that $B$ factors weakly and $T(A)=B$. Then (4.3) clearly entails that $W$ is surjective. It is easy to check that $W^{-1} \in \Gamma(B)$, which in particular shows that $W^{-1}$ is continuous. Now define $\Phi=W^{-1} T$. Of course, $\Phi$ is surjective and continuous. We proceed to show that $\Phi$ is a homomorphism. Let $a, b \in A$. We know that $W(T(a b))=T(a) T(b)$, we have

$$
\begin{aligned}
\Phi(a b) & =W^{-1}(T(a b))=W^{-1}\left(W^{-1}(T(a) T(b))\right) \\
& =W^{-1}\left(T(a) W^{-1}(T(b))\right)=W^{-1}(T(a) \Phi(b)) \\
& =W^{-1}(T(a)) \Phi(b)=\Phi(a) \Phi(b) .
\end{aligned}
$$

Theorem 4.5. Let $A$ be a Banach algebra with an operator space structure and having the property $(\mathbb{B})$ completely and a bounded left approximate identity and suppose that $B$ is a completely contractive Banach algebra. If $T: A \rightarrow B$ is a completely bounded linear map preserving zero products, then there is a closed right ideal $I_{V}$ of $B$ containing $T(A)$ and a bounded linear operator $V: I_{V} \rightarrow B$ such that

$$
\begin{gathered}
\|V\| \leq\|T\| \sup _{i \in I}\left\|\rho_{i}\right\|, \\
V(u v)=V(u) v, \quad V(z w)=z V(w) \quad\left(u \in I_{V}, v \in B, w, z \in \overline{\operatorname{alg} T(A)}\right),
\end{gathered}
$$

and

$$
V(T(a b))=T(a) T(b) \quad(a, b \in A) .
$$

Furthermore the following assertions hold:

(i) If $\overline{T(A)}=B$, then there is $W \in \Gamma(B)$ such that

$$
\|W\| \leq\|T\| \sup _{i \in I}\left\|\rho_{i}\right\| \text { and } W(T(a b))=T(a) T(b)(a, b \in A) .
$$

If $B$ is essential, then $\overline{W(B)}=B$. Moreover, if $B$ is essential and faithful, then $W$ is injective.

(ii) If $B$ is faithful and factors weakly, and $T(A)=B$, then the operator $W$ given in assertion $(i)$ is invertible and there is a continuous epimorphism $\Phi: A \rightarrow B$ such that $T=W \Phi$.

Proof. We define $I_{V}$ and $V$ similar to what we defined in the proof of Theorem 4.4. Since the net $\left(T\left(\rho_{i}\right)\right)_{i \in I}$ is bounded, $I_{V}$ is closed. Existence of bounded left approximate identity for $A$ implies that $A=A^{2}$. Hence $T(A)=T\left(A^{2}\right) \subset I_{V}$ and

$$
V(z w)=z V(w) \quad(w, z \in \overline{\operatorname{alg} T(A)}) .
$$


On the other hand,

$$
\left\|T\left(\rho_{i}\right) u\right\| \leq \sup _{i \in I}\left\|\rho_{i}\right\|\|T\|\|u\| \quad(i \in I) .
$$

Therefore $\|V(a)\| \leq \sup _{i \in I}\left\|\rho_{i}\right\|\|T\|\|a\|$. It shows that the operator $V$ is continuous with $\|V\| \leq\|T\| \sup _{i \in I}\left\|\rho_{i}\right\|$.

Now suppose that $\overline{T(A)}=B$. Since $T(A) \subset I_{V}$ and $I_{V}$ is closed, it follows that $V$ is defined on $B$. Since $V$ is continuous, the operator $W$ given in Theorem 4.4 is $V$.

Corollary 4.6. Let $G$ be a locally compact amenable group. Suppose that B is a completely contractive Banach algebra. If $T: A(G) \rightarrow B$ is a completely bounded surjective linear map preserving zero products, then

(a) If $B$ is faithful and factors weakly, then $T$ is a weighted homomorphism.

(b) $T$ is an epimorphism if and only if $A(G)$ has an approximate identity $\left(\rho_{i}\right)_{i \in I}$ such that $\left(T\left(\rho_{i}\right)\right)_{i \in I}$ is an approximate identity for $B$.

Proof. By Theorem $3.4 A(G)$ has the property $(\mathbb{B})$ completely. Now Part $(a)$ follows from Theorem 4.5 and part $(b)$ follows from Corollary 4.3.

Theorem 4.7. Let $G$ be a locally compact amenable group and suppose that $B$ is a commutative $C^{*}$-algebra or a matrix space. If $T: A(G) \rightarrow B$ is a continuous surjective linear map preserving zero products, then

(a) $T$ is a weighted homomorphism.

(b) $T$ is an epimorphism if and only if $A(G)$ has an approximate identity $\left(\rho_{i}\right)_{i \in I}$ such that $\left(T\left(\rho_{i}\right)\right)_{i \in I}$ is an approximate identity for $B$.

Proof. By Theorem 2.1, $A(G)$ has a bounded approximate identity. Theorem 3.4 shows that $A(G)$ has the property $(\mathbb{B})$ completely. On the other hand all matrix spaces and $C^{*}$-algebras have bounded approximate identities and hence they are faithful and factor weakly. Moreover, being $C^{*}$-algebras, they are completely contractive Banach algebras. [12, Corollary 2.2.2] shows that each linear map from an operator space into a matrix space is completely bounded. [12, Proposition 2.2.6] shows that every bounded linear map from an operator space into a commutative $C^{*}$-algebra is completely bounded. Hence parts $(a)$ and $(b)$ follow from Corollary 4.6(a) and Corollary $4.6(b)$ respectively.

Theorem 4.8. Let $G_{1}$ and $G_{2}$ be two amenable locally compact groups. Suppose that $T: A\left(G_{1}\right) \rightarrow A\left(G_{2}\right)$ is a completely bounded surjective linear map which preserves zero products. Then there are a homomorphism $\Phi: A\left(G_{1}\right) \rightarrow A\left(G_{2}\right)$ and an invertible element $\tau$ in the Fourier-Stieltjes algebra of $G_{2}$ such that

$$
T=\tau \Phi .
$$

Proof. By Theorem 3.4, $A\left(G_{1}\right)$ has the property $(\mathbb{B})$ completely and by Theorem 2.1 , it has a bounded approximate identity. Amenability of $G_{2}$ implies the existence of a bounded approximate identity for $A\left(G_{2}\right)$. Hence $A\left(G_{2}\right)$ is faithful and factors weakly. $A\left(G_{2}\right)$ is a completely contractive Banach algebra. Multiplier algebra of $A\left(G_{2}\right)$ is $B\left(G_{2}\right)$ by Theorem 2.2. Hence

$$
\Gamma\left(A\left(G_{2}\right)\right) \cong Z\left(B\left(G_{2}\right)\right)=B\left(G_{2}\right)
$$


Now Corollary 4.6 implies that there is a homomorphism $\Phi: A\left(G_{1}\right) \rightarrow A\left(G_{2}\right)$ and invertible $\tau$ in $B\left(G_{2}\right)$ such that $T=\tau \Phi$.

Acknowledgement. The author would like to express his gratitude to his advisor Ebrahim Samei for his useful comments.

\section{References}

[1] J. Alaminos, M. Brear, J. Extremera, A. R. Villena, Characterizing homomorphisms and derivations on $C^{*}$-algebras, Proc. Roy. Soc. Edinburg sect. 137 (2007), 1-7. 193 (2009), no. 2, 131-159.

[2] J. Alaminos, M. Brear, J. Extremera, A. R. Villena, Maps preserving zero products, Studia Math. 193 (2009), no. 2, 131-159.

[3] S. Banach, Théorie des opérations linéaires, Warszawa, 1932.

[4] E. Beckenstein and L. Narici, The separating map: a survey, Rend. Circ. Mat. Palermo 52 (1998), 637-648.

[5] M. A. Chebotar, W.-F. Ke and P.-H. Lee, Maps characterized by action on zero products, Pacific J. Math. 216 (2004), 217-228.

[6] M. A. Chebotar, W.-F. Ke and P.-H. Lee, Maps preserving zero Jordan products on hermitian operators, Illinois J. Math. 49 (2006), 445-452.

[7] M. A. Chebotar, W.-F. Ke and P.-H. Lee and N.-C. Wong, Mappings preserving zero products, Studia Math. 155 (2003), 77-94.

[8] M. A. Chebotar, W.-F. Ke and P.-H. Lee and R. Zhang, On maps preserving zero Jordan products, Monatsh. Math. 149 (2006), 91-101.

[9] J. Cui and J. Hou, Linear maps on von Neumann algebras preserving zero products or tr-rank, Bull. Aust. Math. Soc. 65 (2002), 79-91.

[10] H. G. Dales, Banach algebras and automatic continuity, New York, Oxford University Press, 2000.

[11] J. J. Font and S. Hernández, Automatic continuity and representation of certain isomorphisms between group algebras, Indag. Math. (N.S) 6 (1995), 397-409.

[12] E. G. Effros and Z-J Ruan, Operator spaces, New York, Oxford University Press, 2000.

[13] Carl S. Herz, Harmonic synthesis for subgroups, Annales de l'institut Fourier, tome. 23, n 3 (1973), 91-123.

[14] J. Hou and L. Zhao, Zero-product preserving additive maps on symmetric operator spaces and self-adjoint operator spaces, Linear Algebra Appl. 339 (2005), 235244. 
[15] J. Hou and L. Zhao, Jordan zero-product preserving additive maps on on operator algebras, J. Math. Anal. Appl. 314 (2006), 689-700.

[16] W.-F. Ke, B.-R. Li and N.-C. Wong, Zero product preserving maps of operatorvalued functions, Proc. Amer. Math. Soc. 133 (2003), 1979-1985.

[17] V. Losert, On tensor products of Fourier algebra, Arch. Math. 43 (1984), 370-372.

[18] L. Molnár, Selected Preserver Problem on Algebraic Structure of Linear Operators and on Function Space, Lecture Notes in Math. 1895, Springer, Berlin, 2007.

[19] J-P Pier, Amenable locally compact groups, Interscience Publication, New York, 1984.

[20] V. Runde, Lectures on amenability, Lecture Notes in Math. 1774, Springer, Berlin, 2002.

[21] M. Wolff, Disjointness preserving operator on $C^{*}$-algebras, Arch. Math. Soc. 15 (1983), 247-252.

Department of Mathematics and Statistics,

University of Saskatchewan,

106 Wiggins Road,

Saskatoon, SK, CANADA.

email:jas637@mail.usask.ca 